\title{
A rare case of synovial chondromatosis of the temporomandibular joint secondary to discoid lupus erythematosus
}

\author{
Marano R and Araújo S* $^{*}$ \\ Ciências da Cirurgia, Faculdade de Ciências Médicas da Universidade Estadual de Campinas, FCMUNICAMP, Brazil
}

\begin{abstract}
Synovial chondromatosis (SC) is a monoarticular disorder that most commonly affects the large joints. It is rare in the temporomandibular joint (TMJ). When present, however, $\mathrm{SC}$ is typically associated with other conditions. No reports in the literature thus far have described an association between SC and discoid lupus erythematosus (DLE), largely because the latter is predominantly a skin condition. Signs of joints injuries are rare in DLE. This case report describes a case of unilateral TMJ injury in a 46-year-old male patient. After complete removal, through pre-auricular access, the lesion was referred for histological analysis, confirming the diagnosis of EC in the TMJ in a patient with LED. At the moment the patient is in ambulatory follow-up without signs of relapse. In addition, due to the small but risk of recurrence and evolution of discoid and systemic lupus erythematosus, bi-annual monitoring is performed by means of tomography and laboratory tests.
\end{abstract}

\section{Introduction}

Synovial chondromatosis (SC) is a benign arthropathy characterized by chondrometaplasia of the synovial membrane in which cartilaginous nodules form and may become pedunculated and/ or detach from the synovial membrane; in doing so, they become loose bodies within the joint space [1]. SC most commonly involves the large synovial joints-particularly those of the knee, hip, elbow, and ankle. Temporomandibular joint (TMJ) involvement with SC is relatively uncommon $[2,3]$.

The etiology has not been completely clarified. Cases without identifiable etiological factors are referred to as primary SC and are thought to be more aggressive in their behavior. Secondary SC is considered to be associated with slowly progressive degenerative joint diseases, as well as with trauma and neurological diseases [4,5].

Discoid lupus erythematosus (DLE) is the most common subtype of cutaneous lupus erythematosus (CLE): it accounts for $72 \%$ to $83 \%$ of all cases of CLE. The characteristics of DLE have been well described as scaly, erythematous macules and/or papules evolving into scar tissue in the form of hypopigmented plaques with central epidermal atrophy and peripheral hyperpigmentation [6]. Arthralgia/arthritis, changes to nails, anemia, leukopenia, high erythrocyte sedimentation rates, and positive ANA results have all been associated with systemic disease in patients with DLE, regardless of whether the case is secondary to systemic lupus erythematosus (SLE). Even so, systemic manifestations of DLE are rare, and there seem to be no reports in the literature on DLE being associated with SC in any joint, including the TMJ [7].

\section{Case Report}

The patient was a 46-year-old male. Three years prior to his consult, during his routine personal hygiene practices, he injured himself with a razor blade in the forehead region. After the injury healed, the patient noticed that the region remained erythematous. He sought care from a dermatologist and received two incisional biopsies in different regions. The description of the lesion was one of erythematous plaque, approximately $5 \mathrm{~cm}$ in size, forming a round shape on the forehead, with an atrophic and hypochromic central area and hyperchromic and hyperkeratotic margins, and with reduced hair follicle density, a scar-like appearance, and follicular plugs. The histological findings reflected hyperkeratosis, as well as keratin plugs at the openings to the hair follicles, basal cell layer degeneration, and inflammatory lymphocytic infiltrate in the deep connective tissue, all of which were compatible with chronic CLE. The patient was immediately sent for evaluation by a rheumatologist in order to exclude the diagnosis of SLE. Clinically, the patient did not exhibit photosensitivity, oral ulcers, xerophthalmia, xerostomia, Raynaud's syndrome, psoriasis, ocular pathologies, serositis, or other changes compatible with SLE. The laboratory exams were negative for anemia and leukopenia and no abnormalities were found in the urine dipstick chemical analysis, but the exams were positive for antinuclear antibodies (an ANA result of 1:160). Due to the lack of criteria representative of SLE, the patient was treated for DLE. He was prescribed prednisolone sodium (20 mg per day) for topical use and hydroxychloroquine ( $400 \mathrm{mg}$ per day) for oral use. In the same year, the patient experienced pain the region of the left TMJ when opening or closing his jaw. The treatment provided was merely analgesic in nature. After two years, the patient began to experience additional pain, now in the left auricle. He sought care from an otolaryngologist, who ordered an MRI and CT of the region.

*Correspondence to: Araújo S, Ciências da Cirurgia, Faculdade de Ciências Médicas da Universidade Estadual de Campinas, FCMUNICAMP, Brazil, Tel: 55+ (27)996395242; E-mail: stellaaraujo_@hotmail.com

Key words: synovial chondromatosis, temporomandibular joint, discoid lupus erythematosus, articular diseases

Received: June 06, 2018; Accepted: June 19, 2018; Published: June 21, 2018 
The MRI revealed joint effusion with signs of synovitis on the left side associated with a clearly defined tumor. The lesion was oval-shaped, surrounded by a hypoechoic halo sign / hypointense halo sign / a low signal. The inner portion exhibited a heterogenicity compatible with calcifications with a high signal in sequences sensitive to liquids. The dimensions were $2.1 \times 1.0 \times 1.7 \mathrm{~cm}$ (Figures $1 \mathrm{a}, 1 \mathrm{~b}, 2 \mathrm{a}$ and $2 \mathrm{~b}$ ). In the CT, the lesion seemed internally mixed lateral to the left condylar processand exhibited a discretely hypodense halo (Figures 3 and 4). The patient was then sent to an oral and maxillofacial surgeon and underwent surgery to excise the lesion (Figures 5 and 6). The lesion was excised using the preauricular approach due to its dimensions. It was then sent for histopathological analysis, which produced a histological description of the tissue as one having sections with a cartilaginous appearance mixed with synovial tissue with polygonal stromal cells and giant multinucleated cells (Figure 7). The patient is currently receiving outpatient follow-up care from physicians specializing in oral and maxillofacial surgery, dermatology, and rheumatology. He has no functional complaints regarding his TMJ, and there have been no radiographic findings suggestive of cancer relapse. The patient still lacks the criteria necessary for an SLE diagnosis.However, due to the
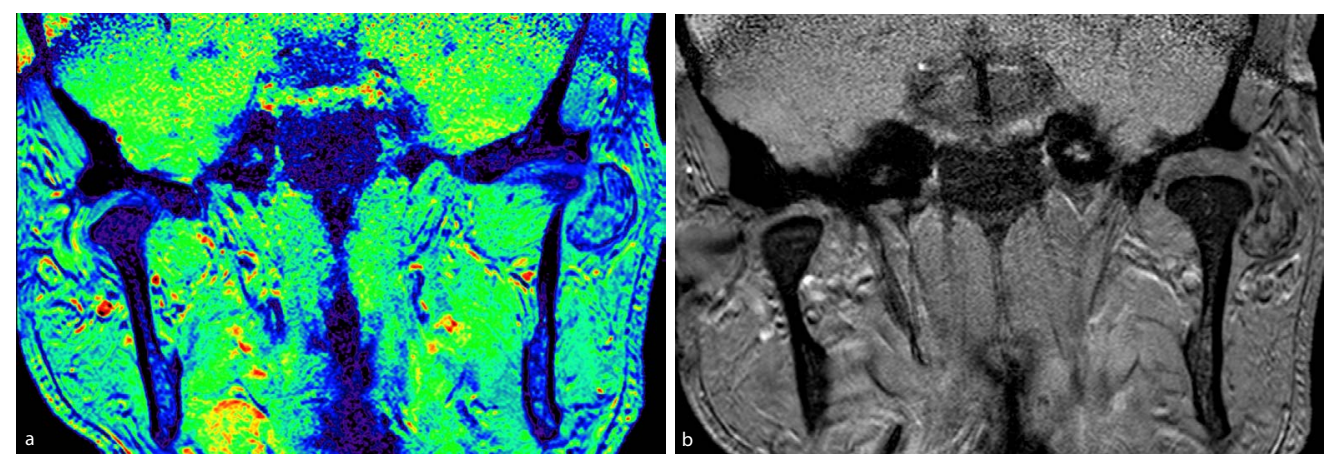

Figure 1a and 1b. Magnetic resonance imaging in a coronal section showing extra-osseous lesion in the left TMJ
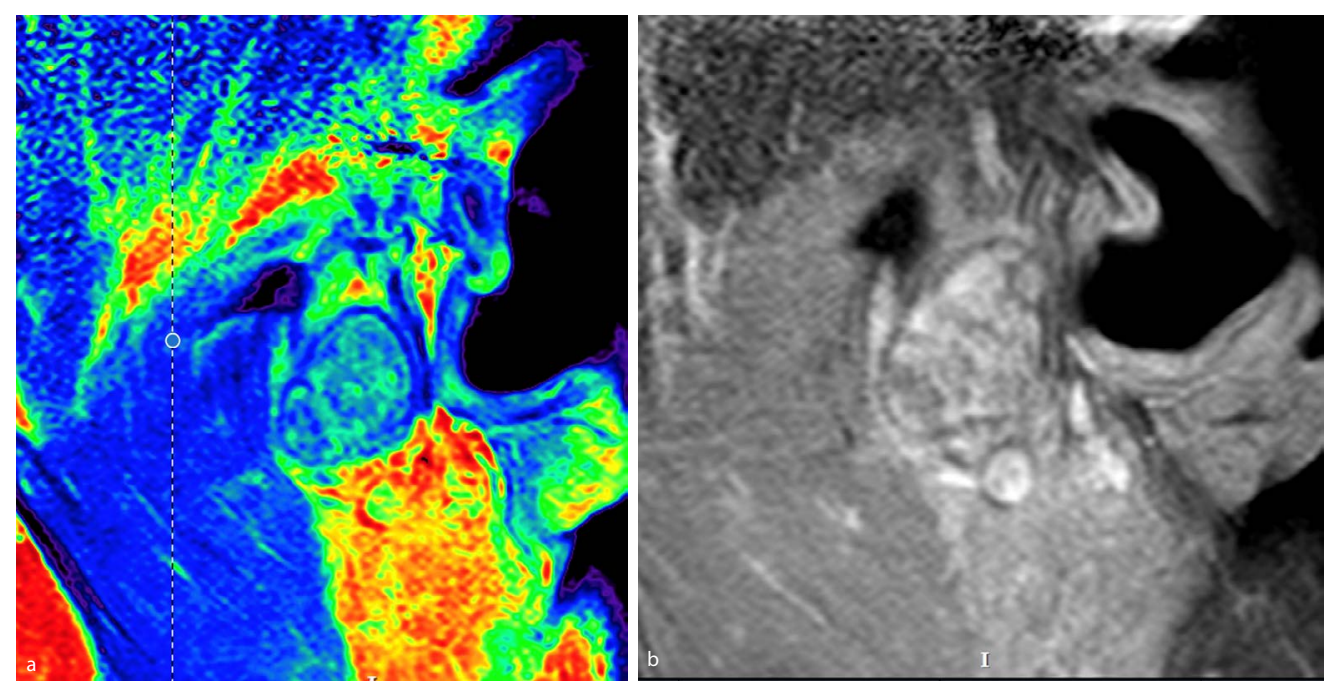

Figure 2a and 2b. Magnetic resonance imaging in a sagittal cut in a left TMJ region showing mixed lesion with hypo and hypersignal

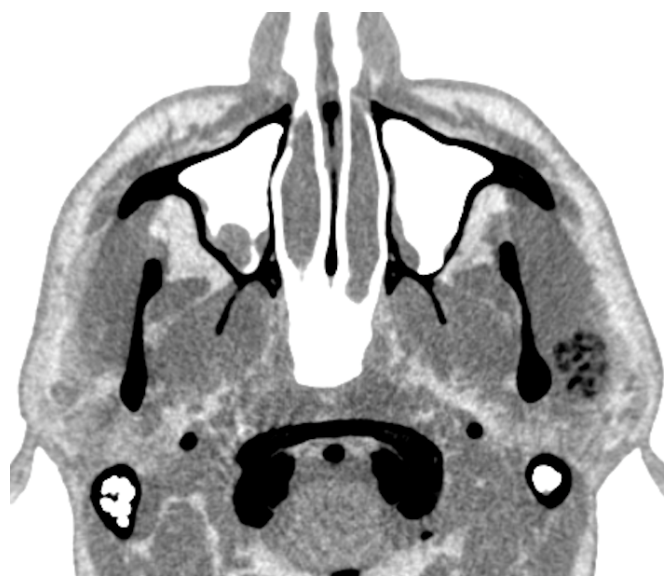

Figure 3. Computed tomography for soft parts inverse in axial section showing lateral lesion to left condylar process 
risk, even if small, of recurrence of the lesion and the evolution of discoid lupus erythematosus to the systemic, a semestral monitoring by means of tomography and laboratory tests is performed.

\section{Discussion}

SC is a rare pathological condition. Though it most commonly occurs in the large joints of the limbs, it also may affect the TMJ. Though there are still relatively few cases in the literature, reports of SC have increased in recent years. This increase may be attributed to improved imaging techniques and increased knowledge on SC of the TMJ [8].

Clinical findings are non-specific in the TMJ. The classic set of symptoms of pain at the site, preauricular swelling, crepitus, and occlusal disturbances is not always present. Other symptoms may include jaw deviation when the mouth is opened, as well as headache and hearing

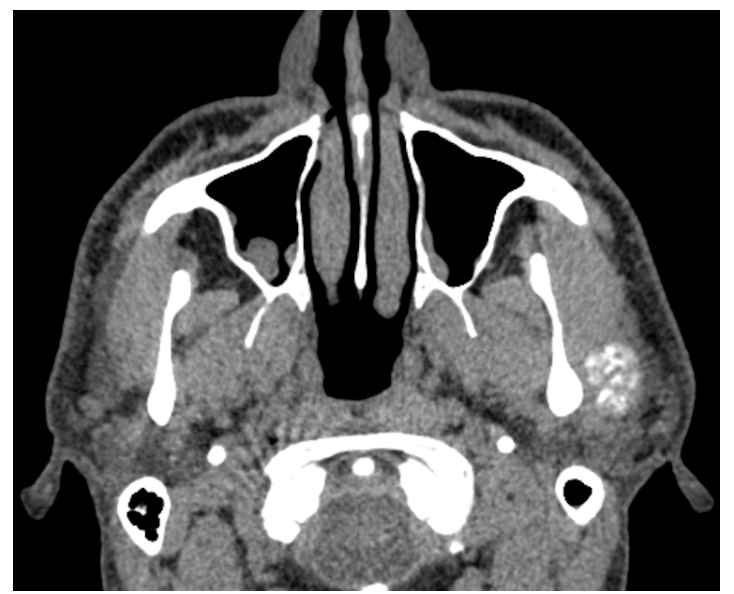

Figure 4. Computed tomography for soft parts conventional in axial section showing lateral lesion to left condylar process

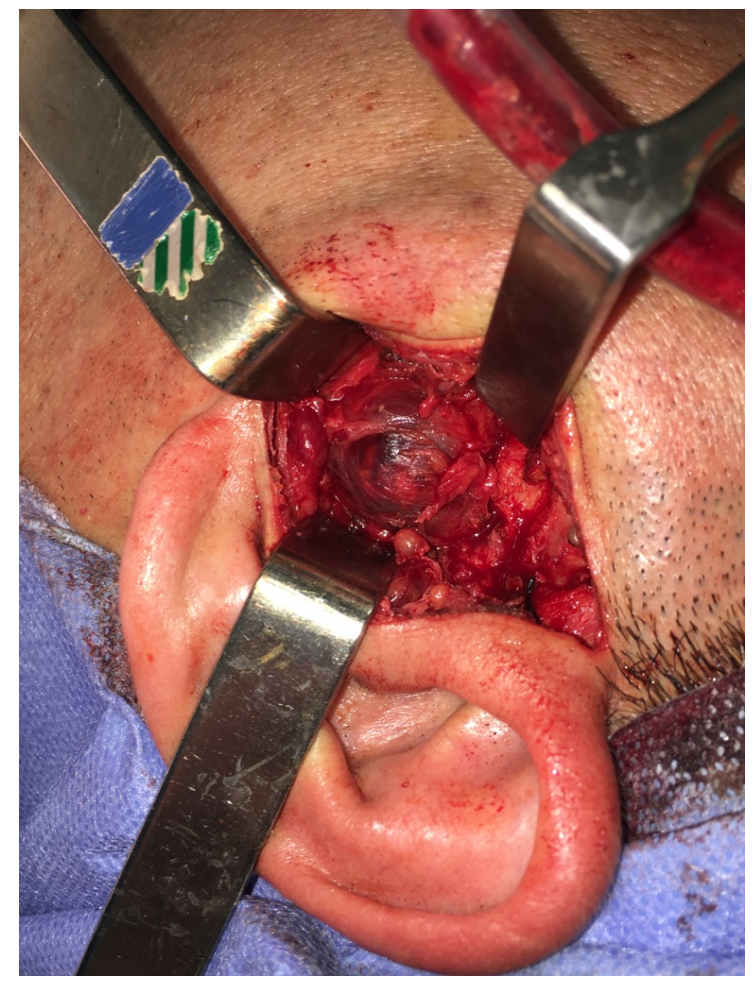

Figure 5. Removal of the lesion through pre-auricular access

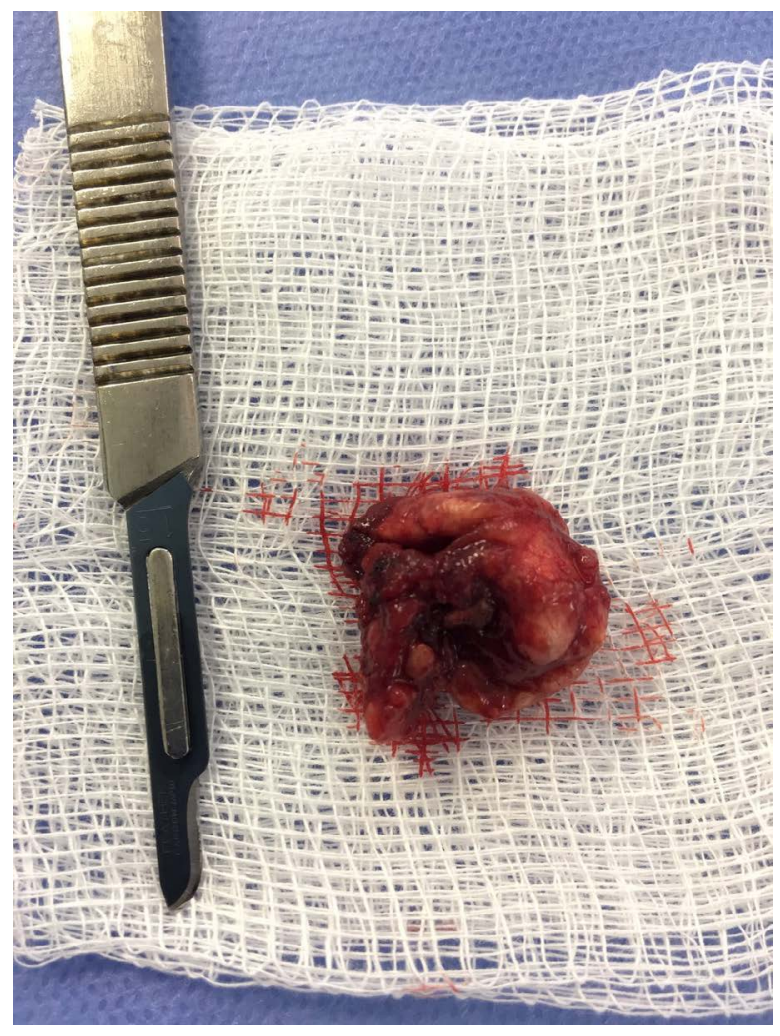

Figure 6. Surgical specimen withdrawn from left TMJ

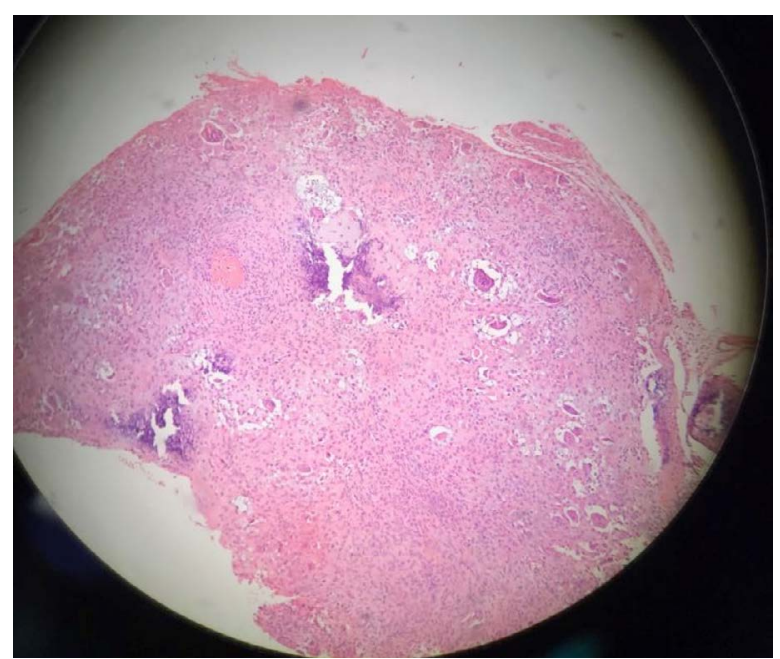

Figure 7. Tissue as one having sections with a cartilaginous appearance mixed with synovial tissue with polygonal stromal cells and giant multinucleated cells

loss. The loose cartilaginous nodules are rarely calcified and thus go undetected during conventional X-ray examination. When combined with the low incidence of the condition in the TMJ, this factor often leads to delayed diagnosis or misdiagnosis [9]. In our case, the patient did not experience arthralgia until the tumor reached considerable dimensions, at which point it generated pain and limited functioning at the site. Since they are able to aid in the precise diagnosis of this type of lesion, MRI and CT have been reported as excellent tools for these cases, particularly given the fact that the cartilaginous nodules present are largely undetectable by conventional radiography [10]. In our case, the combination of CT and MRI provided evidence that was highly 
suggestive of SC, a diagnosis which was confirmed by the histological and immunohistochemical analyses.

The etiology of SC has not been completely clarified. It may be classified as primary, which is rare and has no associated cause, or as secondary, which is more frequently found and which is always associated with another condition, such as osteoarthritis, epiphyseal dysplasia, Legg-Calvé-Perthes disease, aseptic osteonecrosis, rheumatoid arthritis, diabetes, chondrocalcinoses, or SLE $[11,12]$. This case describes the presence of SC in the region of the TMJ in a patient with a diagnosis of DLE, a condition that predominantly exhibits cutaneous manifestations. We were therefore left with the question of how these pathologies were correlated. First, it is important to understand that, though this condition is largely cutaneous in its presentation, up to $28 \%$ of patients with DLE may exhibit systemic manifestations such as telangiectasias, generalized discoid lupus erythematosus, and arthralgia/arthritis, all of which may or may not be associated with SLE, since DLE may progress-albeit slowly (over four months to 34 years)- to SLE [13]. Thus, though there are currently no other reports in the literature of SC in patients with DLE, the possibility of systemic manifestations with this type of lupus leads us to believe that there is a correlation between SC and DLE in the case reported herein. Another question that this case produced was whether this instance of DLE would progress to SLE, as is commonly found in the literature. In Callen's retrospective study of patients with DLE, arthritis was present in all four patients with DLE who progressed to SLE and absent in all 56 DLE-only patients [14]. Insawang et al. found that Thai patients with generalized DLE $(n=54)$ were 2.2 times more likely to develop SLE than their localized DLE counterparts $(n=76)$. These same authors also reported that articular rheumatic disorders in cases of DLE may be found up to 2.7 times more frequently in DLE cases that progress to SLE than in cases of localized DLE $[15,16]$.

As described previously, the presence of arthralgia in cases of DLE has been found to be more common in cases of DLE that progress to SLE. This association is also found when laboratory factors are considered. Findings such as anemia, leukopenia, a high erythrocyte sedimentation rate, and a high ANA result (1:160) are seen more frequently in cases in which DLE progresses to SLE. Millard and Rowell [16] found that all six patients with DLE who progressed to SLE had persistently abnormal laboratory values in hematological and immunological tests, a trend which was not found in any of the patients with DLE whose disease did not spread. However, these numbers cannot be considered absolute. Healy et al, found that, though most cases with high ANA results also involved a correlation between DLE and SLE, two out of 53 DLE-only patients had repeatedly positive ANA test results [17]. Koskenmies, et al. reported that five out of 178 patients with localized DLE exhibited some kind of joint condition [18]. Their study, however, did not describe the types of joint conditions or the joints involved. In another study, Tebbe, et al. evaluated which systemic and laboratory manifestations associated with localized DLE are most suggestive of a progression of DLE to SLE, though this progression does not always occur [19].

They concluded that nephropathy, arthralgia, and high ANA results reflect greater systemic impairment, but the authors note that progression does not necessarily occur when these factors are present, since some cases of localized DLE included systemic manifestations. In a retrospective study on 270 patients in which clinical and immunological findings from subacute cutaneous lupus erythematosus (SCLE) patients were compared to those from DLE patients, Vera-Recabarren et al, reported the presence of arthralgia in both groups, regardless of whether progression to SLE occurred [20]. In the DLE group, twelve of the 158 patients exhibited some kind of arthralgia, and of those twelve, only four had associated cases of SLE. In these same patients' laboratory findings, sixteen non-SLE cases of DLE were associated with positive ANA results. Other studies have also agreed that, though uncommon and more frequently associated with SLE, laboratory abnormalities and clinical findings such as arthralgia may occur in patients with localized DLE [19,21].

In our case, though some systemic and laboratory factors were present, the patient currently does not exhibit sufficient criteria to meet the diagnosis of SLE. Thus, we believe that this is a case of localized DLE with systemic manifestations that may or may not progress to SLE [13]. Regardless of progression status, the presence of SC associated with DLE alone is enough to warrant attention: of the reports in the literature on joint conditions associated with DLE, none describe the presence of SC, a benign arthropathy commonly found in the large synovial joints but rarely in the TMJ [1-5,12,13-18]. Some reports have, in fact, described SC as being associated with SLE, which once again leads us to believe that this case has potencial for progress to SLE [5,22]. Thus, we believe that cases like this should be carefully monitored because the patients may be at risk of developing SLE involvement.

\section{References}

1. Pau M, Bicsák A, Reinbacher KE, Feichtinger M, Kärcher H (2014) Surgical treatment of synovial chondromatosis of the temporomandibular joint with erosion of the skul base: a case report and review of the literature. Int J Oral Maxillofac Surg 43: 600-605. [Crossref]

2. Von Lindern JJ, Theuerkauf I, Niederhagen B, Bergé S, Appel T, et al. (2002) Synovial chondromatosis of the temporomandibular joint: clinical, diagnostic, and histomorphologic findings. Oral Surg Oral Med Oral Pathol Oral Radiol Endod 94: 31-39. [Crossref]

3. Lieger O, Zix J, Stauffer-Brauch EJ, Iizuka T (2007) Synovial chondromatosis of the temporomandibular joint with cranial extension: a case report and literature review. $J$ Oral Maxillofac Surg 65: 2073-2080. [Crossref]

4. Zha W, Zhao YF, Liu Y, Jiang L (2009) A case of synovial chondromatosis of the temporomandibular joint secondary to preauricular trauma. Int J Oral Maxillofac Surg 38: 1212-1215. [Crossref]

5. Suyama Y, Nakayama S, Hagiwara K (2016) Synovial Chondromatosis of the Suprapatellar Pouch of the Knee in a Patient with Systemic Lupus Erythematosus. $J$ Rheumatol 43: 948. [Crossref]

6. Cardinali C, Caproni M, Bernacchi E, Amato L, Fabbri P (2000) The spectrum of cutaneous manifestations in lupus erythematosus- the Italian experience. Lupus 9: 417423. [Crossref]

7. Chong BF, Song J, Olsen NJ (2012) Determining risk factors for developing systemic lupus erythematosus in patients with discoid lupus erythematosus. Br J Dermatol 166: 29-35. [Crossref]

8. Gonzalez-Perez LM, Congregado-Cordoba J, Salinas-Martin MV (2011) Temporomandibular joint synovial chondromatosis with a traumatic etiology. Int J Oral Maxillofac Surg 40: 330.

9. Martínez GR, Torres MP, Mateo MM, García IS, Alba LM, et al. (2011) Bilatera synovial chondromatosis of the temporomandibular joint. J Craniomaxillofac Surg 39: 261-265. [Crossref]

10. Liu, Huang Z, Zhu W, Liang P, Tao Q (2016) Clinical and Imaging Findings of Temporomandibular Joint Synovial Chondromatosis. An Analysis of 10 Cases and Literature Review. J Oral Maxillofac Surg 74: 2159- 2168. [Crossref]

11. Helmy ES, Bays RA, Sharawy MM (1989) Synovial chondromatosis associated with experi- mental osteoarthritis in adult monkeys. J Oral Maxillofac Surg 47: 823-827. [Crossref]

12. Patri B, Mateus C (1999) Ostéochondromatose associée à une ostéoncrose aseptique au cours d'un lupus systémique. La Revue de Médecine Interne 20: 722-723.

13. Wallace DJ, Pistiner M, Nessim S, Metzger AL, Klinenberg JR (1992) Cutaneous lupus erythematosus without systemic lupus erythematosus: clinical and laboratory features. Semin Arthritis Rheum 21: 221-226. [Crossref] 
14. Callen JP (1982) Chronic cutaneous lupus erythematosus - clinical, laboratory, therapeutic, and prognostic examination of 62 patients. Arch Dermatol 118: 412-416. [Crossref]

15. Insawang M, Kulthanan K, Chularojanamontri L (2010) Discoid lupus erythematosus: description of 130 cases and review of their natural history and clinical course. $J$ Clin Immunol Immunopath Res 2: 1-8.

16. Millard LG, Rowell NR (1979) Abnormal laboratory test results and their relationship to prognosis in discoid lupus erythematosus. A long-term follow-up study of 92 patients. Arch Dermatol 115: 1055-1058. [Crossref]

17. Healy E, Kieran E, Rogers S (1995) Cutaneous lupus erythematosus - a study of clinical and laboratory prognostic factors in 65 patients. Ir J Med Sci 164: 113-115. [Crossref]

18. Koskenmies S (2008) Clinical and laboratory characteristics of Finnish lupus erythematosus patients with cutaneous manifestations. Lupus 17: 337-347. [Crossref]
19. Tebbe B, Mansmann U, Wollina U, , Auer-Grumbach P, Licht-Mbalyohere A, et al. (1997) Markers in cutaneous lupus erythematosus indicating systemic involvement. A multicenter study on 296 patients. Acta Derm Venereol 77: 305-308. [Crossref]

20. Vera-Recabarren MA , García-Carrasco M, Ramos-Casals M, Herrero C (2010) Comparative analysis of subacute cutaneous lupus erythematosus and chronic cutaneous lupus erythematosus: clinical and immunological study of 270 patients. $\mathrm{Br} \mathrm{J}$ Dermatol 162: 91-101. [Crossref]

21. Sontheimer RD, Thomas JR, Gilliam JN (1979) Subacute cutaneous lupus erythematosus: a cutaneous marker for a distinct lupus erythemato- sus subset. Arch Dermatol 115: 1409-1415. [Crossref]

22. Loukil H, Frikha F, Snoussi M, Garbaa S, Ben Salah R, Bahloul Z (2015) Ostéchondromatose synoviale de l'articulation carpo-métacarpienne du pouce chez une patiente atteinte d'un lupus érythémateux systémique. Pan Afr Med J 22: 185. [Crossref]

Copyright: $(02018$ Marano R. This is an open-access article distributed under the terms of the Creative Commons Attribution License, which permits unrestricted use, distribution, and reproduction in any medium, provided the original author and source are credited. 